\title{
Imaging in Haboob Lung Syndrome
}

Ragesh Panikkath MD, DNB, DM, Zachary Mulkey MD, Cynthia Jumper MD, MPH

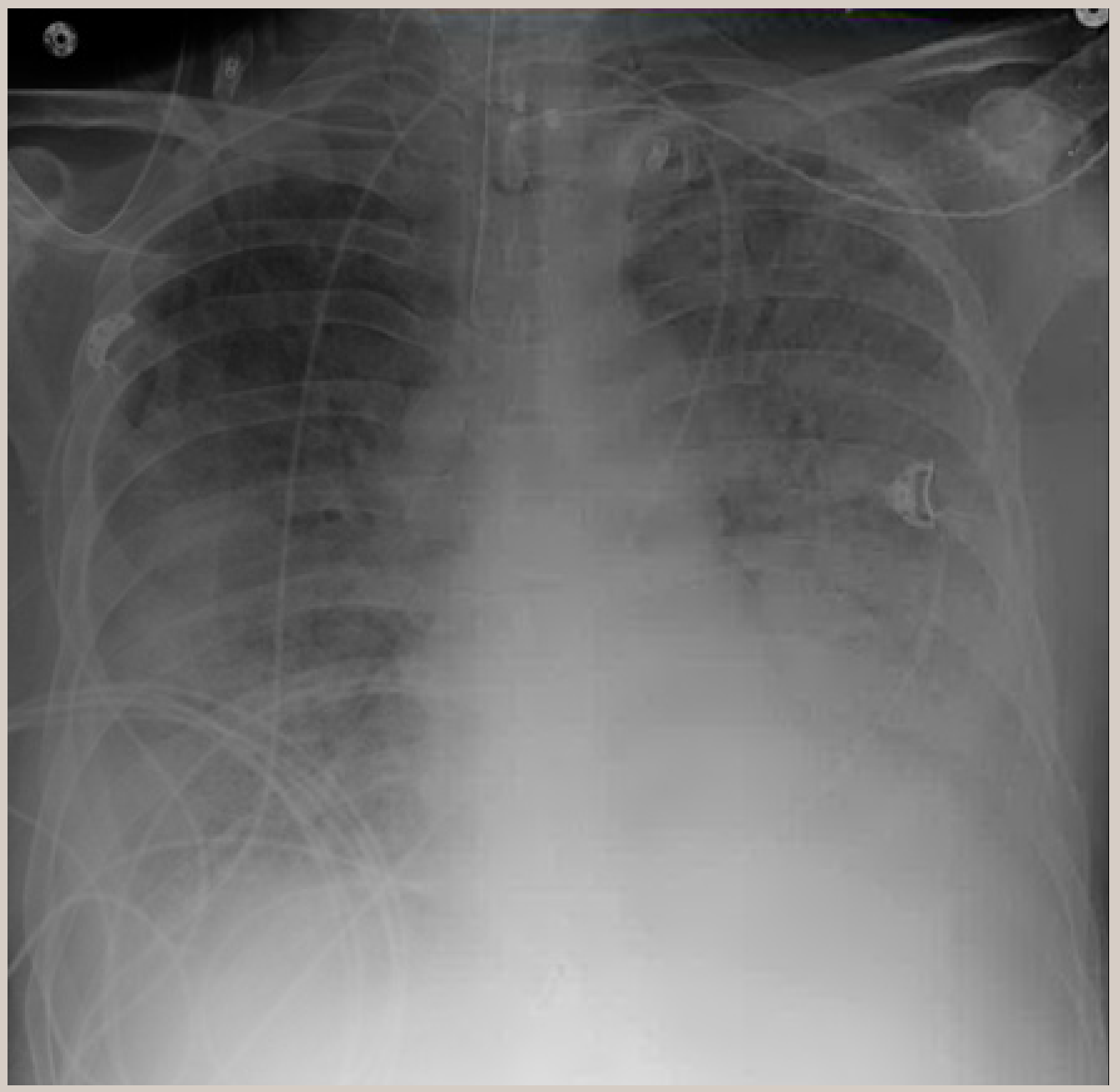

Figure 1 Chest $X$ ray of patient showing multilobar infiltrates after exposure to a dust storm. Also seen is the endotracheal tube, a normal cardiothoracic ratio and no pulmonary venous congestion

There is no medical literature regarding development of acute lung infiltrates/disease after exposure to dust storms except for our recent case series. ${ }^{1} \mathrm{Howev}$ er, pneumonia precipitated by dust exposure occurred

Corresponding author: Ragesh Panikkath Contact Information: ragesh.panikkath@ ttuhsc.edu

DOI: $10.12746 /$ swrccc2013.0102.018 in the dust bowl era (1930s) in the U.S. A retrospective study from Taipei reported an increased incidence of pneumonia on the days following Asian dust storm events. ${ }^{2}$ We present an interesting chest X-ray of a 69-year-old healthy white man taken a few days after exposure to a massive dust storm. ${ }^{3}$ (Figures 1 and 2) He had presented with severe hypoxia and needed mechanical ventilation with $\mathrm{FiO} 2$ of $100 \%$ to maintain a PO2 in high 50s. His chest-X ray showed multilobar infiltrates. His blood, sputum, and bronchoalveolar la- 


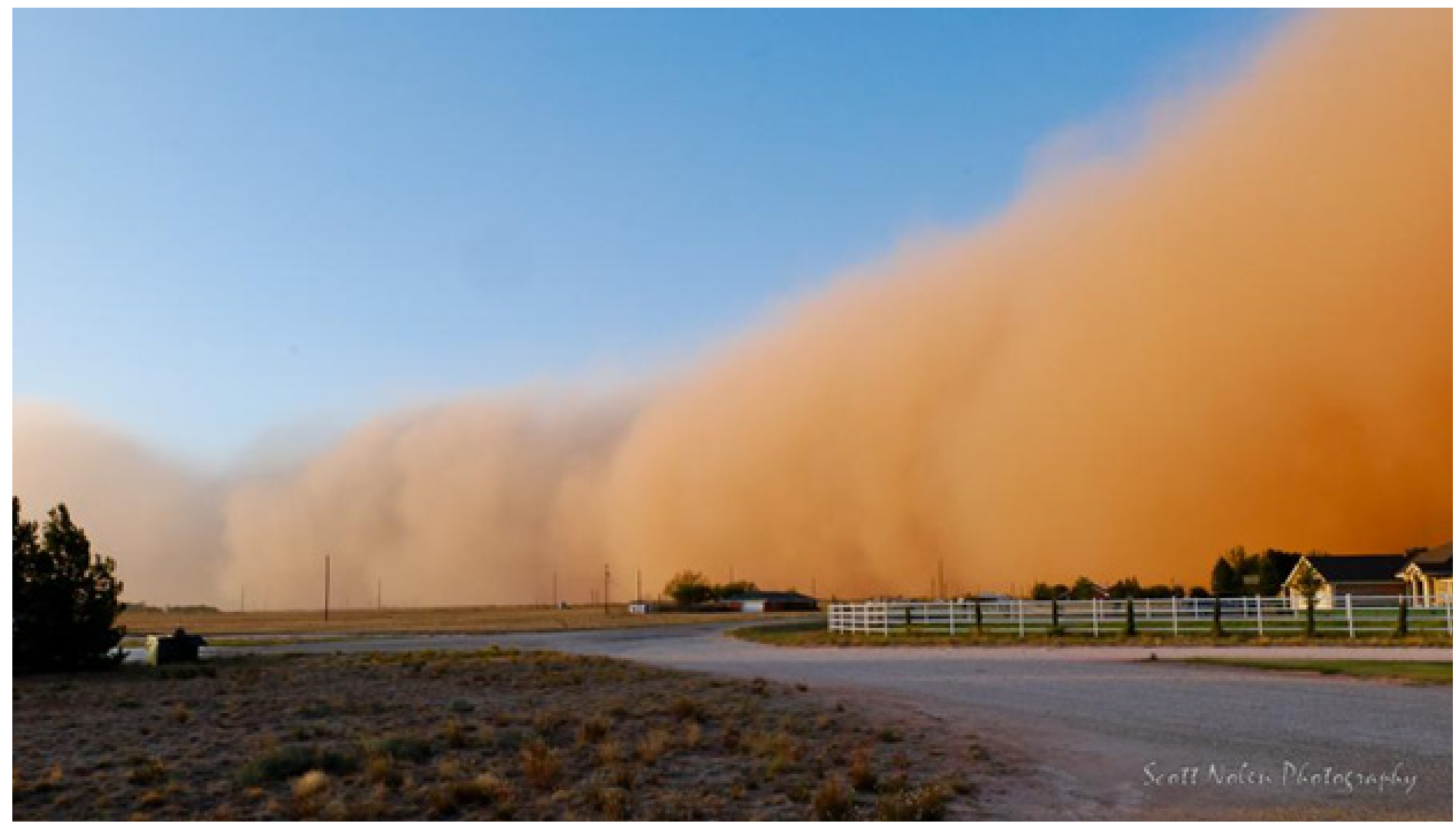

Figure 2 Picture of a dust storm, similar to the one that this patient was exposed to (Image courtesy - Scott Nolen). A video of the actual storm that the patient was exposed to can be seen using this link - http://youtu.be/wfuDFEZYHTE

vage cultures were negative, and he did not respond to empirical broad spectrum antibiotics and antifungals. Legionella antigen, viral studies, and coccidioidomycosis antibody were negative. He expired after a 20 day stormy course in the intensive care unit.

We named this presentation Haboob (Arabic for blasting/drafting) Lung Syndrome, the components being hypoxemia and multilobar infiltrates after exposure to dust storm. ${ }^{1}$ The exact pathogenesis of this syndrome is not clear. Hypersensitivity to dust could be a possible etiology, but this patient did not respond to steroids. A superimposed infection cannot be completely excluded; cultures were negative and the transbronchial biopsy was negative for neutrophils. Prevention seems to be of paramount importance in this condition since the presentation can be severe and life threatening with unclear pathogenesis and treatment options at this time.
Received: $2 / 4 / 13$

Revised: $2 / 8 / 13$

Accepted: 2/10/13

Published online: 4/16/13

Reviewers: Kenneth Nugent MD

Conflict of Interest Disclosures: None

\section{References}

1. Panikkath R, Jumper CA, Mulkey Z. Multilobar lung infiltrates after exposure to dust storm: the haboob lung syndrome. Am J Med. 2013;126:e5-7.

2. Kang JH, Keller JJ, Chen CS, Lin HC. Asian dust storm events are associated with an acute increase in pneumonia hospitalization. Ann Epidemiol. 2012;22:257-63.

3. Dust Storm. wikipedia.com. January 31 2013. http:// en.wikipedia.org/wiki/Dust_storm 